\title{
Evaluation of the Efficacy of Ablative vs. Fractional Er:YAG Laser Modes as a Treatment of Post-Burn Scars
}

\author{
Abdel Rahman Asfour ${ }^{1 *}$, Hisham A Shokeir ${ }^{2}$, Tarek F Elwakil ${ }^{2}$, Fouad M Ghareeb ${ }^{3}$ and Mahmoud S Elbasiouny ${ }^{2}$ \\ ${ }^{1}$ Maadi Plastic Surgery Center, Cairo, Egypt \\ ${ }^{2}$ Medical Applications of Lasers Department, National Institute of Laser Enhanced Sciences, Cairo University, Egypt \\ ${ }^{3}$ Departement of Plastic Surgery, Menofia University, Shebeen El-Kom, Menofia Governorate, Egypt
}

*Corresponding author: Abdel Rahman Asfour, Maadi Plastic Surgery Center, Cairo, Egypt, Tel: +20 2 23599062; E-mail: dr.med.asfour@gmail.com

Received date: March 25, 2017; Accepted date: October 11, 2017; Published date: October 18, 2017

Copyright: (c) 2017 Asfour AR, et al. This is an open-access article distributed under the terms of the Creative Commons Attribution License, which permits unrestricted use, distribution and reproduction in any medium, provided the original author and source are credited.

\begin{abstract}
Aim: To determine the effect of Er:YAG laser in the management of post-burn scars and to compare its effect to ablative and fractional lasers.

Patients and methods: 50 patients with post-burn scars were recruited and randomly divided into 2 groups: Group I patients had ablative Er:YAG laser mode therapy and Group II patients had fractional Er:YAG laser rmode therapy. Vancouver scar score (VSS) was recorded for each group pre- and postoperatively, both groups showed an increase in the VSS and histopathological findings.
\end{abstract}

Results: The obtained results showed morphological changes immediately after treatment, the skin surface displayed a white-gray frost, which on close inspection revealed a point pattern corresponding to individual laser columns done by fractional laser treatment.

Conclusion: Ablative and fractional modes of Er:Yag laser can be used for treatment of post-burn hypertrophic scars. Ablative laser mode had better results than fractional laser mode as indicated by clinical assessment, VSS and changes of histopathological findings.

Keywords: Lasers; Burn scar; Er:YAG; Ablative lasers; Fractional lasers

\section{List of Abbreviations:}

PDL: Pulsed Dye Laser; IPL: Intense Pulsed Light

\section{Introduction}

Post-burn scars have functional and cosmetic influences on affected individuals due to their aberrant wound healing [1]. A healed burn patient may be left with scars and disfigurement which have down effects on self-esteem, body image and overall quality of life [2,3]. Burn scars have, in addition, some functional morbidity such as contractures, hypertrophic changes and keloid formation. Furthermore, burn scars could produce persistent hyperemia, chronic folliculitis, intense and unrelenting pruritis and neuropathic pain $[4,5]$.

Burn scars are either hypertrophic or atrophic scars or keloids; with a number of symptoms and functional deficits. Determining the type of scarring and the associated symptoms is important to decide the type of therapy needed. Treatment of burn scars is challenging and difficult despite the many options available including pressure therapy, silicone gel, intralesional or topical corticosteroids, radiation and interferon [6]. There have been advocates for scar excision, this is usually followed by primary closure, with or without tissue expansion or with flaps or grafts. These therapies have high failure and recurrence rates, as well as significant side effects [7].
For more than 25 years, laser therapy has been used for the treatment of scars; in the medical literature there are different laserand light-based technologies that are poised to dramatically alter our reconstructive algorithm and create a major paradigm shift in the management of burn scars. These are vascular-specific pulsed dye laser (PDL), ablative/non ablative fractional Laser resurfacing, Intense pulsed light (IPL) and some other laser types $[8,9]$. PDL demonstrated an improvement in burn scar texture, pliability, erythema, pruritis, pain and reduction in scar volume (34-66\% improvement) [10].

IPL showed improvement in terms of scar height, erythema and hardness with a moderate level of patient satisfaction although there is lack of evidence for its efficacy [11]. Although the mechanism of action for scar improvements is unknown, most theories are based on the principle that vascular proliferation plays a key role in scar so dye laser and light based therapies could be effective in fresh scars. Mature scars with aberrant collagen deposition are treated with resurfacing. Traditional laser resurfacing is a technique that is commonly accomplished via ablative devices such as conventional carbon dioxide laser, that provides the greatest improvement with a single treatment, but significant adverse effects limit its use and patient downtime can be extensive [12].

Er:YAG lasers, with wavelengths of $2940 \mathrm{~nm}$, are 10 times more selective for water than $\mathrm{CO}_{2}$, laser it penetrates to an average depth of $2-5 \mu \mathrm{m}$ per $\mathrm{J} / \mathrm{cm}^{2}$ and The necrotic layer is completely removed during each new pass, and even after multiple passes, the residual necrotic layer does not exceed 10-15 $\mu \mathrm{m}$. Er:YAG laser is effective in resurfacing skin with fine and superficial atrophic scars, yielding similar results to 
that of $\mathrm{CO}_{2}$ laser, Er:YAG reepithelialization typically takes 4-7 days so it decreases postoperative erythema and recovery times $[13,14]$.

Fractional laser are gaining popularity and have been successfully utilized in the treatment of scars; because fractional resurfacing treating $20 \%, 40 \%$ or $90 \%$ of the area this could provide rapid reepithelialization which consider this treatment highly effective with significant low risk of complications [15]. While normal skin will reepithelialize quickly and evenly from hair follicles and dermal glands after dermabrasion or laser ablation, burn scars are often partially or completely deprived of their epidermal appendages so during resurfacing of such scars, it is advisable to save spots of intact epidermal basal layer, which can serve as islands for reepithelialization [16]. As the Erbium:YAG laser provides ideal options to maintain such reepithelialization procedure.

The aim of this study was to prospectively evaluate the efficacy and safety of a $2940 \mathrm{~nm}$ Er:YAG laser whether in its ablative/fractional modes in the treatment of post burn hypertrophic scars.

\section{Patients and Methods}

This was a prospective study, from both a clinical and histological perspective aspects to study the effect of Er:YAG laser on patients with mature burn scars. The study was conducted in outpatient clinic at the National Institute of Laser Enhanced Sciences, Cairo University, between December 2011 to May 2014. The present study was conducted on 50 patients divided into two groups (I treated with ablative Er:YAG laser mode and II treated with Fractional Er:YAG laser mode) 25 patient each, Gender distribution between groups showed 14 Male (56\%) and 11 Female (44\%) in Group I whereas 13 Male (52\%) 12 Female (48\%) were in Group II at different age groups. The study included mature and stable post burn hypertrophic scars of at least one year duration of different shapes and surface areas and different body locations. This study was approved by local authorities of Cairo University and all subjects provided written informed consents. Patients with keloids tendency, Photosensitivity, below 5 years old or on Oral retinoids within the last 6 months were excluded from the study. The included patients were randomly divided into 2 equal treatment groups: Group-I: included 25 patients subjected to ablative Er:YAG laser. Group-II: included 25 patients subjected to fractional Er:YAG laser.

\section{Laser system}

The laser used in this study was Er:YAG laser (XS dynamics Fotona S1-121d Ljubljana Slovenia) with following specifications $2940 \mathrm{~nm}$ and energy 3J , pulse duration ( $100 \mu \mathrm{s}, 300 \mu \mathrm{s}, 600 \mu \mathrm{s}, 1500 \mu \mathrm{s}$ and $250 \mathrm{~ms}$. (Short Pulse: 300 microsecond SP Mode does not allow heat to be transferred into the tissue and is used when strong ablation is required) and fluence range up to $380 \mathrm{~J} / \mathrm{cmP} 2 \mathrm{P}$.

\section{Technique of ablative mode}

For ablative mode the R11 hand piece was used this, straight hand piece has a variable spot sizes from 2 to $7 \mathrm{~mm}$. The R11 hand piece was used in a freehand method. The single spots were placed slightly overlapping in circles or any other pattern on the skin, while a constant spot overlaping of $30-40 \%$ was maintained. For an even subtotal de epithelialization of larger areas, we applied two passes at energy of $500-1000 \mathrm{~mJ}$ as provided by $5 \mathrm{~mm}$ spot size, an SP mode and $30-40 \%$ spot overlap.
In larger scar areas containing multiple prominent bands and lines, the elevations were first cleared away selectively using high power. In a second step, the whole scar area was sub totally de epithelialized using the above described energy mode in order to obtain an even regeneration and optical blending. On the face, complete aesthetical subunits were treated whenever possible.

\section{Technique for the fractional mode}

The RO4 hand piece offers a unique fractional Er:YAG treatment modality. The RO4 is a variable hand piece that allows the number and size of pixel, as well as the overall spot size, to be varied. The hand piece can be set to provide 7, 10 and $12 \mathrm{~mm}$ treatment spot sizes and Pixel Size 20-300 $\mu \mathrm{m}$, Number of Pixel $4-256$ Pixels according to the selected level. In larger scar areas the technique we did, the first pass on all the area and the second pass only on the elevated bands the average number of passes (3-5), Energy (1000-1200 mJ) Mode SP, Spot size $(7 \mathrm{~mm})$ and frequency $(3-5 \mathrm{~Hz})$. The laser settings were developed from clinical experience with prior scar and resurfacing treatments. Adjustments were made within the described parameters for patient comfort.

\section{Treatment methods}

Patients were treated in the outpatient clinics of NILES, treatment was carried out using a topical anesthetic cream (EMLA [eutectic mixture of lidocaine and prilocaine] AstraZeneca, London, UK) applied to the scar area under occlusion 2 hours before treatment. Immediate follow-up examinations were performed after each session. To evaluate skin improvement, photographs were taken with a digital camera (HD movie 720p, 12.1 megapixels resolution, Sony, Tokyo, Japan) before treatment and at each follow up visit.

\section{Postoperative Care}

Wound care after laser treatment included a topical antibiotic ointment for several days, and return to work within 1 to 3 days. Postoperative analgesia was accomplished with nonsteroidal antiinflammatory agents.

\section{Patient assessments}

Further follow-up was performed 7 and 30 days post treatment to monitor recovery, improvement and any subsequent squeal. Textural scar irregularity was also evaluated by the physician at these time points. The photographs taken before initiation of treatment and 3 months following the end of treatment were independently evaluated and compared.

Side effects and complications were recorded. Before start of treatment all subjects provided written informed consent. Clinical assessment was done before treatment and 6 months after the final treatment, assessment was done using the most widely used assessment scale Vancouver Scar Scale (VSS), which measures vascularity, pliability, pigmentation and height giving a range of $0-14$ in the total score. It was originally designed to rate burn scars as follows vascularity $(0=$ normal, $1=$ pink, $2=$ red, $3=$ purple $), \quad$ Pliability (Normal=0, Flat $=0, \quad$ Supple $=1$, Yielding $=2, \quad$ Firm $=3, \quad$ Ropes $=4$, Contracture $=5)$, Pigmentation $(0=$ normal, 1 =hypo-pigmentation, $2=$ mixed pigmentation, $3=$ hyper-pigmentation) and Height (Flat $=0<2$ $\mathrm{mm}=12-5 \mathrm{~mm}=2>5 \mathrm{~mm}=2$ ). 


\section{Histological assessment}

The biopsy specimens were collected from scars before treatment starts, the area of scar that was biopsied was carefully marked and photographed to ensure having the post treatment biopsy specimens taken adjacent to the pretreatment biopsy. Punch biopsies $3 \mathrm{~mm}$ were performed on the treated areas and sent for tissue processing and staining.

Tissue blocks were fixed in $10 \%$ buffered formalin, embedded in paraffin and sectioned in standard fashion. The stains included hematoxylin-eosin (H\&E) and Masson Trichrome).

\section{Statistical Methods}

Data were collected, tabulated and statistically analyzed using SPSS v. 23 (IBM - Statistical Package for the Social Science). Descriptive statistics was expressed in terms of mean \pm standard deviation $(\mathrm{M} \pm$ SD). Comparison between before and after treatment effect was done using paired sample $t$ test to detect any significant effect between before and after treatment in each group. Unpaired t-test was done to compare the efficacy of both treatments at post treatment assessment stage. $\mathrm{P}$ values less than 0.05 were considered statistically significant $(\mathrm{P}<0.05)$.

\section{Results}

Patients' ages ranged from 9 to 42 years with mean \pm SD 24.0824 .08 \pm 8.001 years for Group I and range of 11-46 years with mean \pm SD $29.57 \pm 7.196$ years for Group II.

Descriptive data of the scars showed a mean \pm SD duration $24.08 \pm$ 199 years for Group I and $13.44 \pm 5.009$ years for Group II. In Group I Patients skin type were $12 \%$ skin type II, $60 \%$ type III and $28 \%$ type IV in Group II $8 \%$ were of skin type II, $68 \%$ type III and $24 \%$ type IV. The distribution of the scars in body areas in Group I were Trunk $28 \%$, Face $36 \%$, Upper limbs $24 \%$ and Lower limbs $12 \%$, and for Group II were trunk $16 \%$, face $32 \%$, upper limbs $32 \%$ and lower limbs $20 \%$ (Table 1).

\begin{tabular}{|c|c|c|c|}
\hline & & $\begin{array}{c}\text { Ablative Er:YAG laser } \\
\text { gp }\end{array}$ & $\begin{array}{c}\text { Fractional Er:YAG laser } \\
\text { gp }\end{array}$ \\
\hline \multirow{2}{*}{ Gender } & Male & $14(56 \%)$ & $13(52 \%)$ \\
\hline & Female & $11(44 \%)$ & $12(48 \%)$ \\
\hline \multirow{2}{*}{ Scar Type } & III & $14(56 \%)$ & $15(60 \%)$ \\
\hline & IV & $11(44 \%)$ & $10(40 \%)$ \\
\hline $\begin{array}{l}\text { Age (mean, } \\
\text { years) }\end{array}$ & & 24.08 & 20.57 \\
\hline \multirow{4}{*}{ Scar Site } & Trunk (T) & $7(28 \%)$ & $4(16 \%)$ \\
\hline & Face $(F)$ & $9(36 \%)$ & $8(32 \%)$ \\
\hline & $\begin{array}{l}\text { Upper Limb } \\
\text { (UL) }\end{array}$ & $6(24 \%)$ & $8(32 \%)$ \\
\hline & $\begin{array}{l}\text { Lower Limb } \\
\text { (LL) }\end{array}$ & $3(12 \%)$ & $5(20 \%)$ \\
\hline $\begin{array}{l}\text { Scar } \\
\text { Duration }\end{array}$ & & $10.88 \pm 5.2$ & $13.44 \pm 5$ \\
\hline
\end{tabular}

Table 1: Descriptive statistics results.
The clinical assessment of the scars revealed that most scars had increased pigmentation compared with surrounding noninvolved skin. Hypertrophy was noted in some treatment areas. Immediately after treatment, the skin surface displayed a white-gray frost, which on close inspection revealed a pinpoint pattern corresponding to fractional laser dots. Assessment before each session after month of procedure and then every month revealed complete healing within 10-12 days and the scar surface was smooth and has soft texture (Figures 1-3).

The obtained results showed that data were differed in pre and post treatment within each group however between the two groups the results showed dramatic differences for traditional ablative Er:YAG laser treatment over fractional Er:YAG laser treatment group the obtained results were for traditional ablative Er:YAG laser, the descriptive data results expressed as mean \pm SD revealed that for assessment results $6.64 \pm 1$ and $4.7 \pm 1.2$ for pre and post treatment, respectively. The obtained results revealed high significant differences between pre and post treatment as $p$ value $=0.0001$. For fractional Er:YAG laser, the descriptive data results expressed as mean $\pm \mathrm{SD}$ revealed that for assessment results $7.77 \pm 0.7$ and $6.4 \pm 0.99$ for pre and post treatment, respectively. The obtained results revealed high significant differences between pre and post treatment as $p$ value $=0.0001$. Unpaired t-test was done to compare the efficacy of both treatments at post treatment assessment stage. The obtained results showed that data were significantly differed in post treatment between both groups $\mathrm{p}=0.0001$, however the results showed dramatic differences for Traditional ablative Er:YAG laser treatment over Fractional Er:YAG laser treatment group the obtained results were as mean difference (1.94) for traditional was greater than that of the fractional laser group (1.3). Regarding studying the effect of scar duration, skin type, age, gender and scar site on the treatment efficacy in both groups, the obtained results showed that pretreatment there was no significant effect on treatment efficacy as $p=0.389$ and 0.95 for traditional ablative Er:YAG and fractional Er:YAG respectively. Also in post-treatment there was no significant effect as $p=0.849$ and 0.994 for traditional ablative Er:YAG and fractional Er:YAG, respectively. Regarding the effect of skin type, the results showed in pretreatment there was no significant effect on treatment efficacy as $p=0.795$ and 0.933 for traditional ablative Er:YAG and fractional Er:YAG, respectively. Also in post-treatment, there was no significant effect as $\mathrm{p}=0.99$ and 0.858 for traditional ablative Er:YAG and fractional Er:YAG respectively. Regarding effect of age, the results showed in pretreatment there was no significant effect on treatment efficacy as $p=0.498$ and 0.231 for traditional ablative Er:YAG and fractional Er:YAG, respectively; but in post-treatment assessment age showed high significant effect as $p=0.015$ as good results obtained within the range of 16-20 years, age showed no significant effect on treatment evaluation for fractional Er:YAG as $\mathrm{p}=0.364$. Regarding the effect of gender, the results showed in pretreatment there was no significant effect on treatment efficacy for traditional ablative Er:YAG and fractional Er:YAG as $p>0.05$. Regarding effect of scar site, the results showed in pretreatment there was slight significant effect on treatment efficacy for traditional ablative Er:YAG and fractional Er:YAG as $\mathrm{p}=0.037$ and 0.047 , respectively. The results showed in post-treatment there was no significant effect on treatment efficacy for traditional ablative Er:YAG and fractional Er:YAG as p $>0.05$. 
Citation: $\quad$ Asfour AR, Shokeir HA, Alwakil TF, Ghareeb FM, Elbasiouny M (2017) Evaluation of the Efficacy of Ablative vs. Fractional Er:YAG Laser Modes as a Treatment of Post-Burn Scars. Biol Med (Aligarh) 9: 415. doi:10.4172/0974-8369.1000415

Page 4 of 6

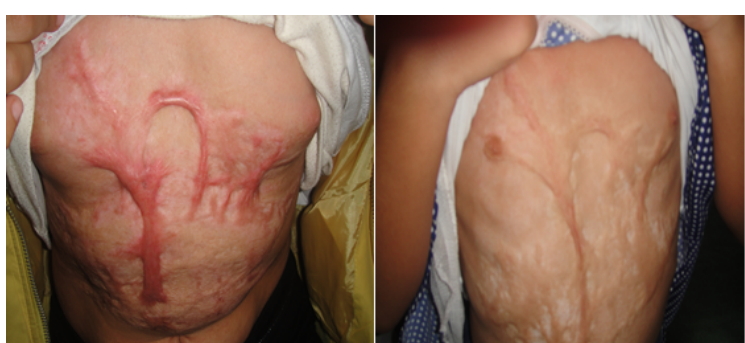

Figure 1: Post burn scar in the trunk area treated by fractional laser change of height and pliability.

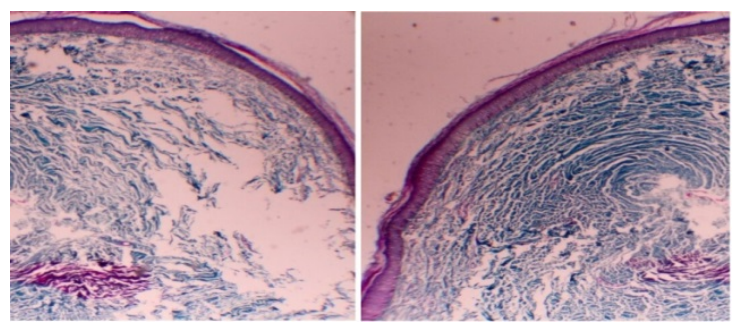

Figure 2: Pre- and post-ablative laser histopathological findings by Masson trichrome stain: post burn biopsy by Masson trichrom $\mathrm{x}$ 200 show epidermal hyperplasia, hyperkeratosis, flat ridges and papilomatosis; ablative Er-Yag laser by masson trichrom $\times 200$ show decrease thickness with flat rete ridge in epidermas.

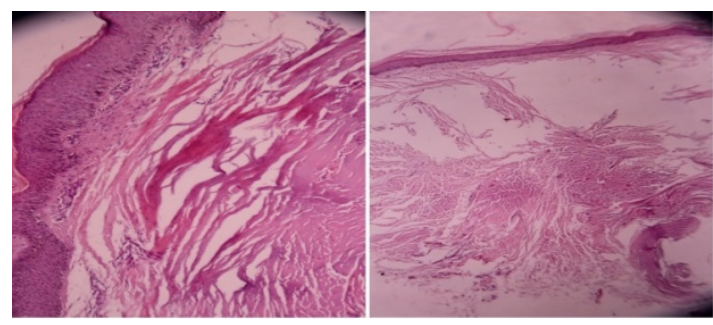

Figure 3: Pre- and post-ablative laser histopathological findings by H\&E stain: post burn biopsy by H\& E x 200 show epidermal hyperplasia hyperkeratosis, flat ridges and papilomatosis; ablative Er-Yag laser by H\&E x 200 show decrease thickness with flat rete ridge in epidermas.

\section{Results of Vancouver Scar Assessment Scale}

Comparison by Paired samples t-test between pre and post treatment mean \pm SD values of (VSS) of Group I (traditional ablative headpiece) was compared and revealed high significant differences between pre and post treatment as the mean value of VSS total score post treatment was decreased to 4.7 as compared to 6.64 VSS total score pretreatment $(\mathrm{p} \leq 0.0001)$. For Group II (fractional headpiece) there was significant decrease between the mean \pm SD VSS total score $7.77 \pm 0.7$ pretreatment to a mean \pm SD $6.4 \pm 0.99$ post treatment $(\mathrm{p} \leq$ 0.05 ). In order to compare post treatment results between both groups, unpaired t-test was done to compare the efficacy of both treatments at post treatment assessment stage. The obtained results showed that data were significantly differed in post treatment between both groups $\mathrm{p}=0.0001$, as the results showed dramatic differences for Traditional ablative Er:YAG laser treatment over Fractional Er:YAG laser treatment group the obtained results were as mean difference (1.94) for traditional was greater than that of the fractional laser group (1.3) (Table 2).

\begin{tabular}{|l|l|l|l|}
\hline \multicolumn{1}{|c|}{ Group } & \multicolumn{1}{|c|}{ Status } & \multicolumn{1}{c|}{ Mean } & \multicolumn{1}{c|}{ Std. Deviation } \\
\hline Fractional Er:YAG laser & Pre-treatment & 7.77 & 0.687 \\
\cline { 2 - 4 } & Post-treatment & 6.40 & 0.995 \\
\hline $\begin{array}{l}\text { Traditional ablative Er:YAG } \\
\text { laser }\end{array}$ & Pre-treatment & 6.64 & 1.003 \\
\cline { 2 - 4 } & Post-treatment & 4.70 & 1.243 \\
\hline
\end{tabular}

Table 2: Treatment effect assessment in pre and post treatment in ablative and fractional groups.

The comparison for Vancouver Scar Scale VSS results among the two groups for pre and post-treatment assessment the data were analyzed using nonparametric test (Mann-Whitney Test) to detect any significant differences between the two groups, the obtained results showed that comparing the results in pre and post revealed highly significant differences for pigmentation, vascularity, pliability and height as $\mathrm{p}=0.0001$. If we compare both groups as two different treatment modalities (ablative and fractional) we found that the obtained results showed highly significant differences in posttreatment on contrary of pretreatment, as $p=0.004,0.01,0.0001$ and 0.005 for vascularity, pliability, pigmentation and height respectively as shown in Figure 4 . All results in pretreatment comparison between the two groups showed no significant difference as $\mathrm{p}>0.05$.
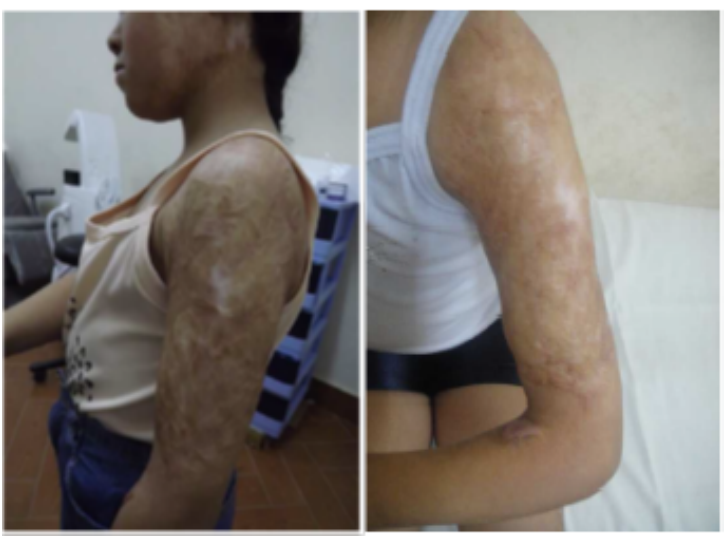

Figure 4: Post burn scar in upper limb treated by ablative laser change of height and pliability. 


\section{Histopathological Results}

\section{Pretreatment histopathology of burn scar samples stained with H\&E}

The epidermis was characterized by flattening of the rete ridges, hyperkeratosis, hypergranulosis and regular palisading basal cell layer, blood vessels were oriented vertically.

The dermis showed nodules composed of aggregates of fibroblasts, small vessels, thicker and stretched collagen bundles were seen throughout the dermis. A low-grade inflammation in the dermis in the form of lymphocytes around telangiectatic vessels was found.

In Masson's trichrome stained samples replacement of papillary dermis with abnormal hyperplastic thicker collagen bundles was noticed and the peripheral layers of collagen forming a septal-like capsule.

\section{Post treatment histopathology of Burn scar samples stained with $\mathrm{H} \& \mathrm{E}$}

a-The epidermis showed improved appearance after Er:YAG laser as the keratinocytes become well organized and malpighian layer thickness had increased together with thinning in the stratum corneum.

b-The dermis showed a remarkable histological finding as cellular infiltrates was found in the upper dermis with increased number of fibroblasts and increased dermal vascularity. Masson Trichrome stain revealed well-organized collagen bundles in the papillary dermis parallel to the epidermis with compact appearance, the only difference between samples after ablative and after fractional laser is that the ablative laser samples showed more parallel and dense collagen bundles also a horizontal oriented fibrillar collagen. There was evident neocollagen formation in both samples.

\section{Discussion}

Conventional treatment of post burn scar was surgical but it is one of the most difficult challenges facing reconstructive surgeons, and improving the shape of cicatrix itself becomes a good option. Dermabrasion was firstly used; however, despite many case reports in literature, it is unclear if the carbon dioxide laser or the erbium laser alone provides a long-term significant improvement. More recently, fractionated resurfacing both non ablative and ablative have been shown to have some effect on subsets of burn scars [16].

In this study, the clinical efficacy of Er:YAG laser in the treatment of post burn scars was investigated. This was found to provide highly controlled ablation with only minimal thermal necrosis, even after multiple passes [17]. In post burn scars, adnexal structures are usually destroyed and spontaneous healing can arise from the surrounding healthy skin that may results in delayed wound healing, while $\mathrm{CO} 2$ lasers seem to be more effective for smoothing scars it goes along with delayed healing time depending on the wound size [18]. We consider the Erbium:YAG laser to be more suitable for the treatment of scars due to lesser thermal necrosis [19].

Fractional Er:YAG laser offers a significant increase in depth of treatment and at the same time, enlarges safety margin due to substantial volume of tissue remaining intact [20]. To our knowledge, no other studies compared the ablative versus fractional modes of Er:YAG laser. In this study, patients with burn hypertrophic scars were selected for treatment with Er:YAG laser and assessed using VSS where the following parameters were assessed each one alone and for the total score (vascularity, pliability, pigmentation and height). Er:YAG laser generates improvements in post burn scarring as VSS assessments indicated that for about 6-11 treatments performed, on average, 24.5 days apart resulted in clinically and statistically significant improvement in Group I as VSS total score post treatment was decreased to $4.7 \pm 1.2$ as compared to $6.64 \pm 1$ VSS total score pretreatment $(\mathrm{p} \leq 0.0001)$ and for there was significant decrease between the mean \pm SD VSS total score $7.77 \pm 0.7$ pretreatment to a mean \pm SD $6.4 \pm 0.99$ posttreatment $(\mathrm{p} \leq 0.05)$. These results were in accordance to results from previous studies clinical improvement was seen in all profile treatments of larger areas in the face, neck, lower neckline and hands showed improvement and they concluded, that Erbium:YAG laser to be a valuable supplementary tool for the improvement of cosmetically disturbing mild post burn hypertrophic scars [21]. The major criticism for that study is the authors had no subjective score to assess the scar improvement and it is just a translation of author's experience. Another study investigated the Er:YAG laser for surgical and post traumatic scar showed Clinical improvement in scars according to investigator assessment: $40 \%$ of patients had excellent improvement of $76-100 \%$ (grade 3), 50\% of patients had good improvement of $50-75 \%$ (grade 2), $10 \%$ had fair improvement of 26-49\% (grade 1) at three month follow up.

It is noteworthy to state that the treatments with Er:YAG laser improved the existing pigmentations from burn scarring where the ablative Er:YAG mean \pm SD $1.60 \pm 0.500$ decreased to $0.32 \pm 0.557$ posttreatment and for fractional Er:YAG decreased from $1.84 \pm 0.374$ to $0.96 \pm 0.841$ post-treatment. Also it did not induce additional PIH. The percentage of subjects with skin types IV included in this study is limited due to pigmentation concerns. Even with the use of hydroquinone pre- and post-treatment, a recently published prospective study of 15 subjects with skin types IV-VI and acne scarring using Er:YAG laser showed a 50\% rate of the PIH [22].

The results of the present and previous studies highlight several important issues as the duration of the postoperative recovery and incidence of prolonged erythema may be lower with Er:YAG laser skin resurfacing than with $\mathrm{CO}_{2}$ laser resurfacing, also transient post inflammatory hyperpigmentation is common and may last significantly longer for the ablative handpiece than that seen after fractional Er:YAG laser; however, it may not be as persistent as that experienced after $\mathrm{CO}_{2}$ laser resurfacing. Areas with hypopigmentation showed no response to treatment. Last, the average clinical improvement seen following Fractional Er:YAG laser treatment for burn scars is slightly less that seen after ablative Er:YAG laser and less than $\mathrm{CO}_{2}$ laser resurfacing. This finding is in agreement with previous clinical and histologic studies comparing the effect of high-energy $\mathrm{CO}_{2}$ and Er:YAG lasers resurfacing $[23,24]$.

\section{Conclusion}

In conclusion, Er:YAG laser is a safe and effective modality for the treatment of post burn hypertrophic scarring. The fractional Er:YAG lasers offer an advantage over ablative Er:YAG resurfacing by effecting better postoperative recovery due to rapid re- epithelization and less danger of long-lasting side effects but also less scar improvement. Additional conformational studies are warranted to assess Ablative vs. fractional lasers treatments ablative Er:YAG laser treatments. 
Citation: $\quad$ Asfour AR, Shokeir HA, Alwakil TF, Ghareeb FM, Elbasiouny M (2017) Evaluation of the Efficacy of Ablative vs. Fractional Er:YAG Laser Modes as a Treatment of Post-Burn Scars. Biol Med (Aligarh) 9: 415. doi:10.4172/0974-8369.1000415

Page 6 of 6

\section{Declarations}

This work was conducted under ethical approval from the Faculty of Medicine, Cairo University. Written consent was obtained from patients involved in this study. Consent for publication of all individuals is available on request.

\section{Competing Interests}

The authors declare there is no conflict of interests.

\section{Authors' Contribution}

All authors contributed equally to writing and methodology presented in this work.

\section{Acknowledgments}

The authors are grateful for the Egyptian Musculoskeletal Research Association. (EMRA) for their help in improving writing and editing this manuscript. Grateful thanks and profound respect to Prof. Soliman Saba, Professor of histopathology, Department of pathology, Cairo University and to Prof. Dalai Abdelfatha, Professor of histopathology, Department pathology, Menoufia University, for their supervision. In addition, we would like to thank Dr Osama Fekry Ahmed (Associate Professor of Photobiology, National Laser Institute, Cairo University).

\section{References}

1. Ozyurt K, Colgecen E, Baykan H, Ozturk P, Ozkose M (2012) Treatment of superficial cutaneous vascular lesions: Experience with the long-pulsed $1064 \mathrm{~nm}$ Nd:YAG laser. The Scientific World Journal.

2. Bock O, Schmid-Ott G, Malewski P, Mrowietz U (2006) Quality of life of patients with keloid and hypertrophic scarring. Arch Dermatol Res 297: 433-438.

3. Van Loey NE, Van Son MJ (2003) Psychopathology and psychological problems in patients with burn scars: epidemiology and management. Am J Clin Dermatol 4: 245-272.

4. Berman B, Viera MH, Amini S, Huo R, Jones IS (2008) Prevention and management of hypertrophic scars and keloids after burns in children. J Craniofac Surg 19: 989-1006.

5. Hultman CS, Edkins RE, Lee CN, Calvert CT, Cairns BA (2012) Shine on: Review of laser-and light-based therapies for the treatment of burn scars. Dermatology research and practice.

6. Alster TS, Tanzi EL (2003) Hypertrophic scars and keloids: etiology and management. Am J Clin Dermatol 4: 235-243.
7. Tavares Filho JM, Belerique M, Franco D, Porchat CA, Franco T (2007) Tissue expansion in burn sequelae repair. Burns 33: 246-251.

8. Parrett BM, Donelan MB (2010) Pulsed dye laser in burn scars: Current concepts and future directions. Burns 36: 443-449.

9. Hædersdal M, Moreau KE, Beyer DM, Nymann P, Alsbjørn B (2009) Fractional nonablative $1540 \mathrm{~nm}$ laser resurfacing for thermal burn scars: a randomized controlled trial. Lasers Surg Med 41: 189-195.

10. Vrijman C, Van Drooge AM, Limpens J, Bos JD, Van Der Veen JP, et al. (2011) Laser and intense pulsed light therapy for the treatment of hypertrophic scars: A systematic review. Br J Dermatol 165: 934-942.

11. Bouzari N, Davis SC, Nouri K (2007) Laser treatment of keloids and hypertrophic scars. Int J Dermatol 46: 80-88.

12. Thomas JR, Somenek M (2012) Scar revision review. Archives of facial plastic surgery 14: 162-174.

13. Majaron B, Srinivas SM, Huang HE, Nelson JS (2000) Deep coagulation of dermal collagen with repetitive Er:YAG laser irradiation. Lasers Surg Med 26: 215-222.

14. Nouri K, Vidulich K, Rivas MP (2006) Lasers for scars: A review. J Cosmet Dermatol 5: 14-22.

15. Saryazdi S, Mohebbi A (2012) Evaluation of fractional CO2 laser efficacy in acne scar. J Lasers Med Sci 3: 56.

16. Tanzi EL, Alster TS (2002) Treatment of atrophic facial acne scars with a Dual-Mode Er:YAG laser. Dermatol Surg 28: 551-555.

17. Gladstone HB, Berg D, McDonald M (2010) Scar revision. Dermatol Res Pract.

18. Weinstein C (1998) Computerized scanning Erbiumi: YAG laser for skin resurfacing. Dermatol Surg 24: 83-89.

19. Kwon SD, Kye YC (2000) Treatment of scars with a pulsed Er:YAG laser. Cutan Laser Ther 2: 27-31.

20. Waibel J, Graber E, Davis S, Badiavas E (2012) Effects of erbium fractional resurfacing on third degree hypertrophic burn scars. Lasers in Surgery and Medicine 44: 11-11.

21. Dierickx C, Khatri K, Altshuler G, Erofeev A, Smirnov M, et al. (2007) Fractionated delivery of Er:YAG laser light to improve efficacy and safety of ablative resurfacing procedure. Lasers Surg Med.

22. Eberlein A, Schepler H, Spilker G, Altmeyer P, Hartmann B (2005) Erbium: YAG laser treatment of post-burn scars: potentials and limitations. Burns 31: 15-24.

23. Mahmoud BH, Srivastava D, Janiga JJ, Yang JJ, Lim HW, et al. (2010) Safety and efficacy of erbium-doped yttrium aluminum garnet fractionated laser for treatment of acne scars in type IV to VI skin. Dermatol Surg 36: 602-9.

24. Manuskiatti W, Iamphonrat T, Wanitphakdeedecha R, Eimpunth S (2013) Comparison of fractional erbium-doped yttrium acne scars in Asians. Dermatol Surg 39: 111-20. 